\title{
Distribuição espacial de pivôs centrais no Brasil: I - Região Sudeste
}

\author{
Wulf Schmidt ${ }^{1}$, Rubens D. Coelho ${ }^{2}$, Marco A. Jacomazzi ${ }^{3}$ \& Mauro A. H. Antunes ${ }^{4}$
}

1 Bolsista do CNPq (Foto)

2 DER/ESALQ/USP. CEP 13418-900, Piracicaba, SP. Fone: (19) 3429-4217. Bolsista pesquisador CNPq. E-mail: rdcoelho@esalq.usp.br

3 Bolsista da CAPES. E-mail: majacoma@esalq.usp.br

4 UFRRJ, Departamento de Engenharia Rural. CEP 23851-970, Rio de Janeiro, RJ. E-mail: mantunes@ufrj.br

Protocolo $89-13 / 5 / 2003$ - Aprovado em 2/4/2004

\begin{abstract}
Resumo: Quando se buscam, em literatura, dados sobre a irrigação no Brasil, o que se encontra disponível é a área irrigada por estado e por método de irrigação. A distribuição dentro dos estados, o número de equipamentos e seu tamanho são de difícil obtenção, mesmo junto às indústrias. No entanto, essas são informações fundamentais quando se pretende estudar a disponibilidade hídrica ou o potencial de crescimento da área irrigada de uma região, por exemplo, temas esses bastante atuais com a crescente demanda por água potável em nível mundial e nacional. Com o presente trabalho objetivou-se coletar essas informações utilizando-se de imagens do satélite Landsat com base na característica intrínseca do sistema de pivô central de marcar com um círculo o local onde está instalado. Em função do referenciamento cartesiano das imagens e da sua articulação com os mapas do IBGE, foi possível desenhar os mapas estaduais com as áreas de concentração desse tipo de equipamento que, quando comparados com outras fontes, permitiram concluir-se pela validade da metodologia para obtenção dessas informações.
\end{abstract}

Palavras-chave: pivô central, mapas, irrigação

\section{Spatial distribution of center pivots in Brazil: I - Southeast Region}

\begin{abstract}
Irrigated areas by state and by irrigation method is the available information about irrigation in Brazil. Distribution inside the states, number or size is not easily found even through the industry. These data are very important when one intends to study water availability or growth potencial of irrigated areas for a specific site due to the increasing concern about drinking water worldwide and nationally. The present study had the objective to collect such information using satellite images from Landsat based on the intrinsic characteristic of the center pivot equipments, the circular mark on landscape easily visible. With comparison the geo-referenced images and its articulation with the official IBGE maps, it was possible to draw the state maps with the equipment density distribution, which, when compared to other available sources permitted to validate the methodology used for this purpose.
\end{abstract}

Key words: center pivot, maps, irrigation

\section{INTRODUÇÃO}

O uso racional da água é, sem dúvida, o assunto do momento, devido principalmente à crescente demanda hídrica e sua escassez. O gerenciamento dos recursos hídricos para se evitar conflitos é, pois, fundamental, e para que ele ocorra de maneira eficiente, são necessárias informações sobre as várias fontes de demanda em nível de microbacias. No entanto, para a agricultura irrigada as informações disponíveis mencionam apenas a área irrigada por sistema por estado, sem conter informações sobre a sua distribuição geográfica no estado. Este dado é fundamental para estudos de disponibilidade hídrica em uma microbacia específica, por exemplo, ou ainda estudos de viabilidade para ampliação do perímetro irrigado.

O sistema de irrigação tipo pivô central chegou ao Brasil na década de 1970, mas apenas nas décadas seguintes se consagrou como sistema de irrigação, impulsionado sobretudo por programas governamentais como o PROINE, PROFIR e o PRONI. De fácil operação, alta eficiência no uso da água (70 a $90 \%$ segundo Christofidis, 2002) e baixo custo operacional, os 
pivôs rapidamente conquistaram a preferência do agricultor irrigante.

Quando se pergunta aos fornecedores de equipamentos sobre qual o número de pivôs instalados no Brasil, não há uma resposta precisa. A página da Valmont (Valmont, 2002) na Internet, por exemplo, relata em 10.000 o número desses equipamentos instalados, mas eles mesmos reconhecem ser esse dado uma estimativa. Mesmo em relação à área irrigada por esse tipo de equipamento, há divergências em função da fonte consultada. A fonte atualmente mais aceita é Christofidis (2001, 2002), cujos dados para o Brasil e região sudeste são apresentados na Tabela 1.

Tabela 1. Evolução das áreas irrigadas (ha) por pivô central no Brasil e na região sudeste por estado

\begin{tabular}{lcrrr}
\multicolumn{1}{c}{ Região } & 1996 & 1998 & 1999 & \multicolumn{1}{c}{2001} \\
\hline Brasil & 519.090 & 550.262 & 561.843 & 651.548 \\
Região Sudeste & 336.000 & 349.830 & 354.580 & 362.618 \\
Espírito Santo & 6.000 & 12.100 & 12.060 & 13.688 \\
Minas Gerais & 80.000 & 85.420 & 87.950 & 87.950 \\
Rio de Janeiro & - & 210 & 210 & 6.620 \\
São Paulo & 250.000 & 252.100 & 254.360 & 254.360 \\
\hline
\end{tabular}

Objetivando complementar a informação disponível, o presente trabalho visa não apenas determinar o número de equipamentos instalados mas, também, a sua distribuição geográfica nos estados.

\section{MATERIAL E MÉTODOS}

As imagens utilizadas para este levantamento provêm do satélite Landsat 7/ETM+ e, eventualmente, Landsat 5 TM, disponibilizadas pela Embrapa (2002). As imagens foram obtidas na época seca (Tabela 2) quando a nebulosidade é reduzida (buscou-se menos que $10 \%$ de nebulosidade), fato que facilita o trabalho, reduzindo os erros associados à análise.

Tabela 2. Intervalo de datas da obtenção das imagens pelo satélite, por estado

\begin{tabular}{lcc}
\hline \multicolumn{2}{c}{ Estado } & Irtervalo \\
\hline Espírito Santo & $7 / 5 / 2001$ & $17 / 7 / 2001$ \\
Minas Gerais & $10 / 4 / 2001$ & $10 / 9 / 2001$ \\
Rio de Janeiro & $30 / 5 / 2001$ & $10 / 9 / 2001$ \\
São Paulo* & $23 / 3 / 2001$ & $19 / 8 / 2001$ \\
\hline * Usou-se uma imagem de 1/11/1999 pela sua melhor qualidade
\end{tabular}

Por outro lado, a diferenciação entre a área do pivô e as áreas vizinhas é em alguns casos, prejudicada em virtude de estarem em fase de preparo de solo.

As imagens estão disponíveis nas escalas de 1:500.000; $1: 250.000 ; 1: 100.000 ; 1: 50.000$ e 1:25.000, mas que se optou por fazer a contagem pela menor escala $(1: 25.000)$ porém os mapas foram confeccionados tomando-se como base a escala de 1:50.000, múltiplo da escala utilizada pelo IBGE (1:500.000) para confecção dos mapas estaduais facilitando, deste modo, a confecção dos mesmos. Nesta escala, cada foto representa um quadrado com 15 min cartesianos de lado e uma área aproximada de $700 \mathrm{~km}^{2}$.
Cada imagem apresenta uma identificação que permite a sua navegabilidade através do mosaico, onde cada nível de identificação corresponde a uma escala. Este fato foi utilizado para a confecção dos mapas. Por exemplo, a sigla SF, na foto identificada como SF-23-V-C-V-4, significa a linha paralela ao equador crescente em sentido ao pólo. A passagem do satélite no sentido longitudinal é representada por 23 , crescente em direção a Greenwich. SF-23 divide-se em quatro quadrantes: V, $\mathrm{X}, \mathrm{Y}, \mathrm{Z}(1: 500.000)$; em que cada um, por sua vez, se divide em novos quatro: A, B, C e D (1:250.000); esses, por outro lado, se dividem em seis: I, II, III, IV, V e VI (1:100,000); que novamente se dividem em quatro 1, 2, 3 e 4 (1:50.000), e, por fim, se dividem em NO, NE, SE e SO (1:25.000).

As contagens foram feitas diretamente das imagens disponibilizadas e apenas para aquelas com menor resolução ou com maior dificuldade, utilizaram-se recursos de CAD, para melhor visualização.

\section{RESULTADOS E DISCUSSÃO}

A totalização do número de pivôs para a região sudeste pode ser visualizada na Tabela 3 . Ao se dividir a área irrigada por pivô por estado (Christofidis, 2002), pelo número de pivôs obtido para obter-se a área média por equipamento, assim, identificar-se distorções, uma vez que não existem pivôs com área irrigada acima de 160 ha (São Paulo e Rio de Janeiro, Tabela 3). Para explicá-las, é necessário se conhecer como as informações sobre as áreas irrigadas foram obtidas. Normalmente, essas informações são obtidas junto às indústrias e/ou sua associação (ABIMAQ-Associação Brasileira das Indústrias de Máquinas), porém a própria indústria questiona esses números; do cadastro das outorgas de uso de água concedidas; dos dados do IBGE; da FAO e de outros órgãos. Infelizmente, essas informações apresentam erros associados, por exemplo à empresa adquirente do equipamento, cuja sede jurídica é em São Paulo ou no Rio de Janeiro, mas o local de instalação do equipamento é em outro estado. Se o levantamento de área for baseado no endereço de fatura constante nas notas fiscais, cria-se o tipo de distorção observada na Tabela 3.

As contagens em si, também estão sujeitas a uma possibilidade de erro, função da metodologia utilizada, que se acredita não ser superior a $5 \%$. Os principais fatores que incidem sobre a margem de erro, são: resolução da imagem, nebulosidade, sobreposição de imagem (cada imagem sobrepõe em torno de $10 \%$ em cada direção), tamanho do pivô (os menores são mais difíceis de serem visualizados) e falta de contraste entre a área irrigada e as áreas vizinhas. Na Figura 1A apresenta-se uma foto com boa resolução da região de Casa Branca, SP, enquanto

Tabela 3. Número aferido de pivôs na Região Sudeste*

\begin{tabular}{lrrc}
\hline \multicolumn{1}{c}{ Região } & $\begin{array}{r}\text { Total } \\
\text { pivôs }\end{array}$ & Área (ha)* & $\begin{array}{c}\text { Área média por } \\
\text { equipamento (ha) }\end{array}$ \\
\hline Sudeste & 4.134 & 362.618 & 87,72 \\
Espírito Santo & 329 & 13.688 & 41,60 \\
Minas Gerais & 2.485 & 87.950 & 35,39 \\
Rio de Janeiro & 15 & 6.620 & 441,33 \\
São Paulo & 1.305 & 254.360 & 194,91 \\
* Segundo Christofidis, (2002). & &
\end{tabular}


na Figura 1B uma outra, com menor clareza no município de Votuporanga, SP, caso em que a deficiência se deve ao fato das áreas estarem em fase de preparo de solo.

Para se avaliar a precisão da metodologia utilizada, comparouse a distribuição obtida com algumas informações disponíveis como, por exemplo, um mapa do município de Casa Branca,SP,datado de 1998 e elaborado pelo Instituto de Pesquisa Tecnológica - IPT, o qual mostrava a localização e o tamanho dos pivôs existentes naquele momento no município. Independentemente do tamanho dos pivôs, através da metodologia proposta nesse levantamento conseguiu-se localizar a totalidade dos pivôs assinalados no mapa, demonstrando a sua acuracidade.

Em nível estadual utilizou-se um mapa com a distribuição do número de outorgas de água concedidas em Minas Gerais (ITEM, v.52/53, 2001/2002, p.69) para Minas Gerais, e um mapa com a distribuição do número de agricultores irrigantes usuários de pivôs, feito pela CATI (Coordenadoria de Assistência Técnica Integral) em São Paulo. Em ambos os casos, há uma sobreposição significativa entre as áreas de concentração de pivôs aferidas por este levantamento e as áreas de concentração das outorgas, $\mathrm{MG}$, ou de usuários,SP; tal fato permite a validação do método para esse tipo de levantamento. As Figuras de 2 a 5 mostram a distribuição e o número de pivôs identificados para os estados de Minas Gerais, São Paulo, Espírito Santo e Rio de Janeiro, respectivamente.

As figuras quantificam as informações disponíveis, pois para Minas Gerais (Figura 2) as áreas de maior concentração de pivôs são as regiões de Paracatu, Patos de Minas, Montes Claros, Araguari, Uberaba e Uberlândia. Para São Paulo (Figura 3), as regiões são: Guaíra, o eixo que vai de Casa Branca a São João da Boa Vista, a região de Paranapanema (Holambra
A.

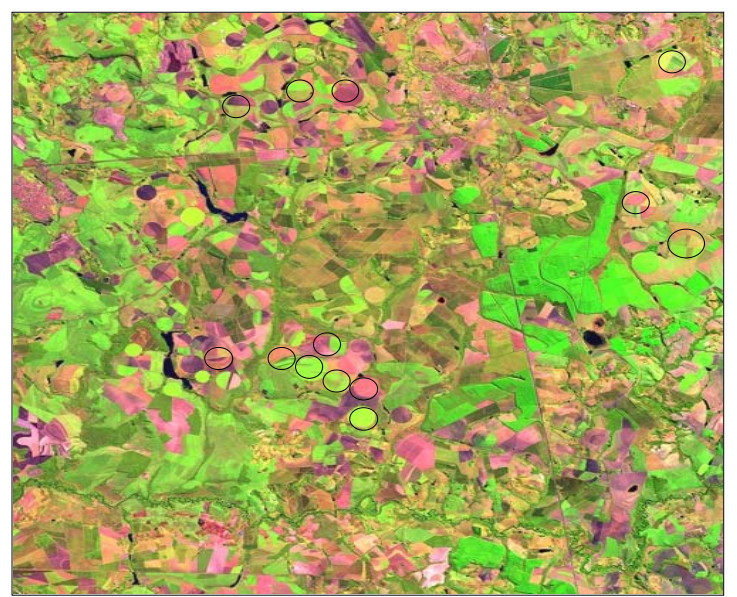

B.

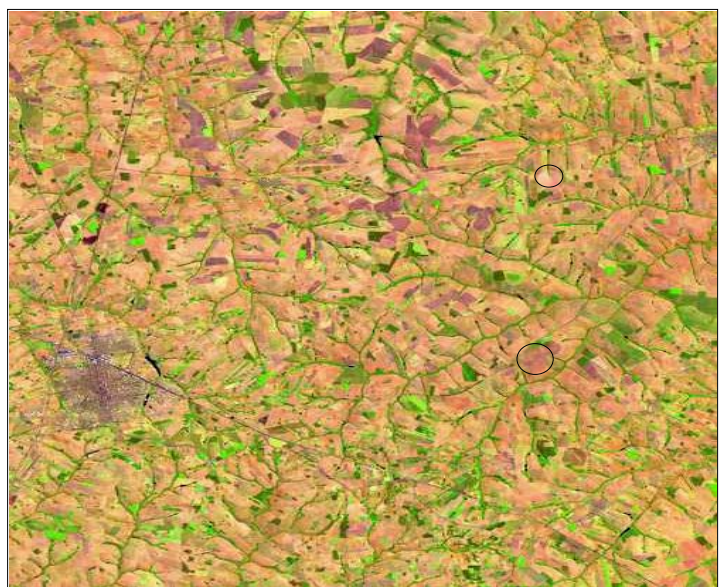

Figura 1. Diferenças na qualidade das imagens: A) Imagem com boa resolução na região de Casa Branca,SP (SF-23-V-C-V-4), e B) Imagem com resolução deficiente na região de Votuporanga/SP (SF-22-X-A-III-3)

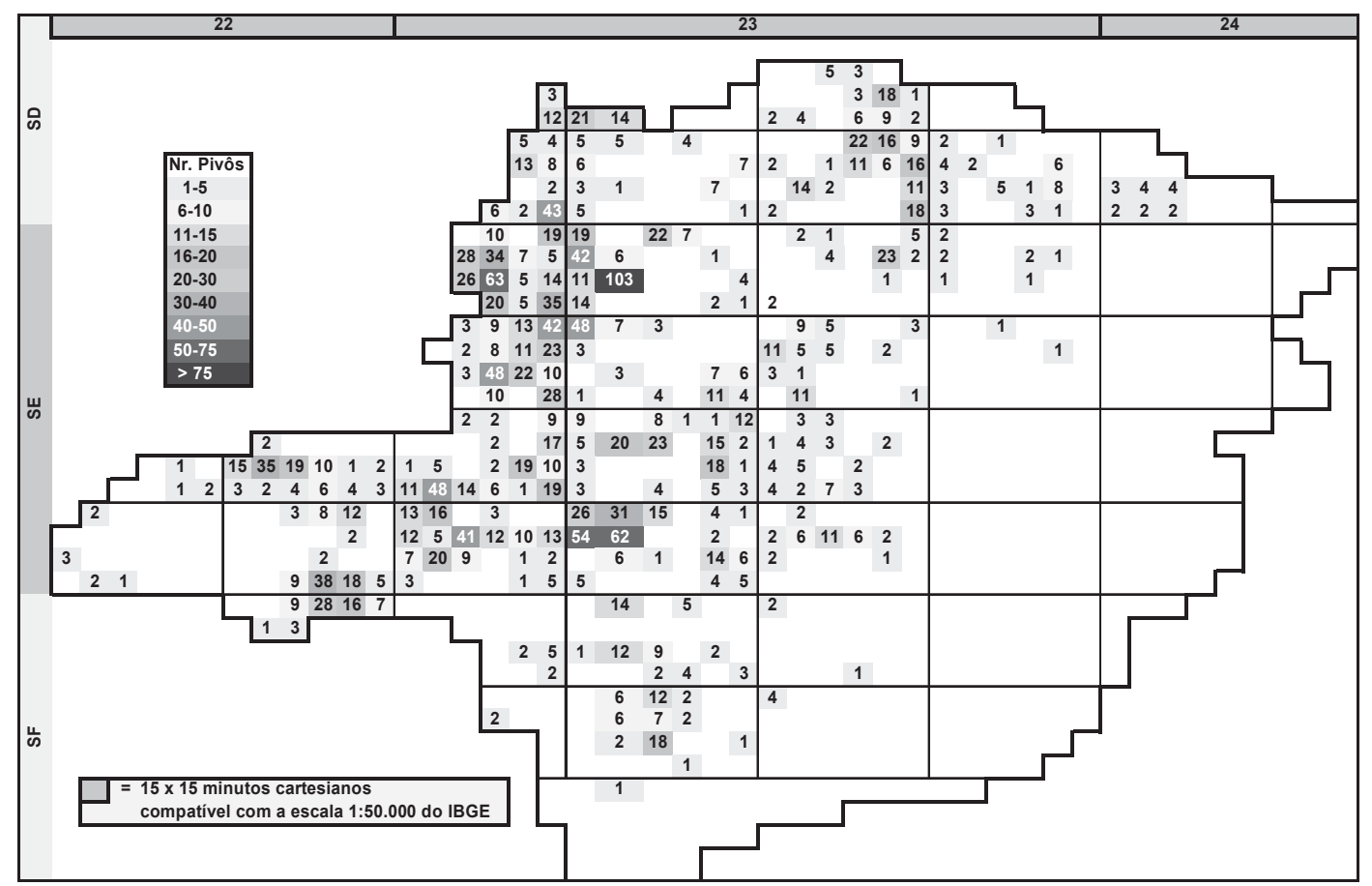

Figura 2. Distribuição espacial dos 2485 pivôs obtidos para Minas Gerais 


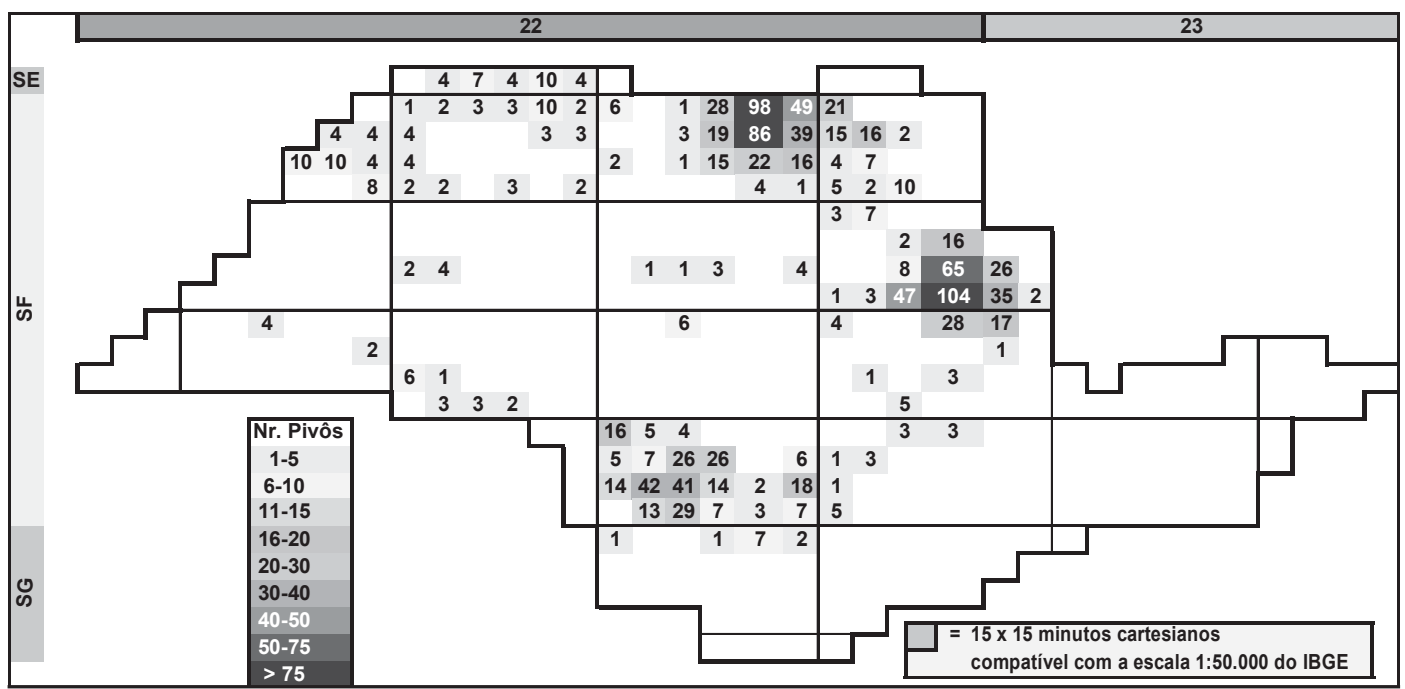

Figura 3. Distribuição espacial dos 1305 pivôs obtidos para São Paulo

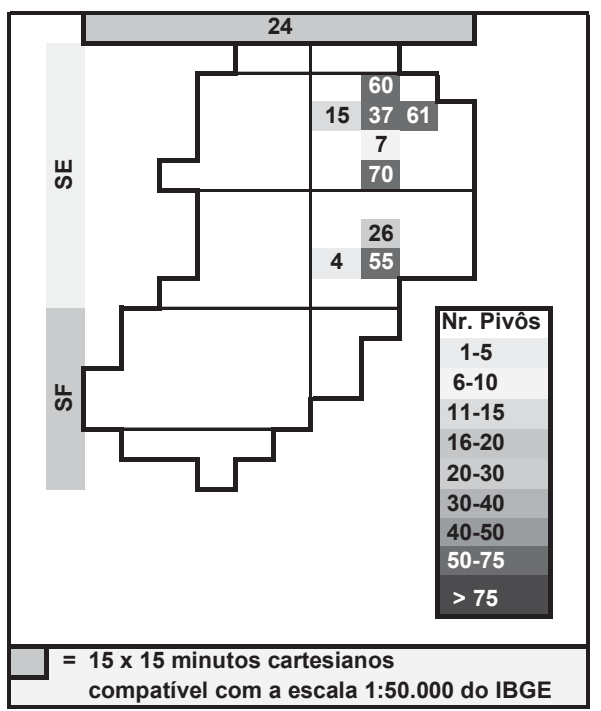

Figura 4. Distribuição espacial dos 329 pivôs obtidos para o Espírito Santo

II) e Votuporanga. Para o Espírito Santo (Figura 4) as regiões de Linhares (Bacia do Rio Doce) e de São Mateus (Bacia do Rio São Mateus) e, finalmente, no Rio de Janeiro (Figura 5), a região de Campos (Bacia do Paraíba) e a região de Macaé (Bacia dos Rios Capivari e São João).

Pode permitir nos levantamentos futuros, a metodologia e obter informações imprescindíveis ao respeito da distribuição por tamanho, para se poder aferir a área efetivamente irrigada, e georreferenciada de modo a permitir acessar o número e a distribuição por tamanho em nível de município ou de microbacias.

\section{CONCLUSÃO}

Pelos dados apresentados, pode concluir-se que a metodologia utilizada se mostrou válida para a determinação do número e distribuição de equipamentos de irrigação tipo

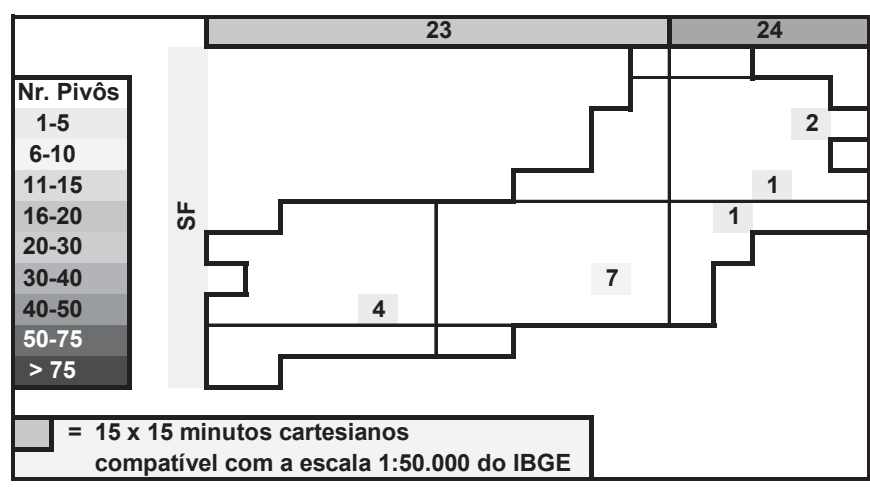

Figura 5. Distribuição espacial dos 15 pivôs obtidos para o Rio de Janeiro

pivô central complementando, deste modo, as informações disponíveis em outras fontes.

\section{LITERATURA CITADA}

Christofidis, D. Os recursos hídricos e a prática da irrigação no Brasil e no mundo. Irrigação e Tecnologia Moderna, Brasília, n.49, p.8-13, 2001.

Christofidis, D. Irrigação, a fronteira hídrica e a produção de alimentos. Irrigação e Tecnologia Moderna, Brasília, n.54, p.46-55, 2002.

Coordenadoria de Assistência Técnica Integral - CATI www.cati.sp.gov.br, 12 out 2002.

Miranda, E.E. (coord.) Espírito Santo In: O Brasil visto do espaço. EMBRAPA Informação Tecnológica, Brasília/DF, CD-Rom, 2002.

Miranda, E.E. (coord.) Minas Gerais In: O Brasil visto do espaço. EMBRAPA Informação Tecnológica, Brasília/DF, CD-Rom, 2002.

Miranda, E.E. (coord.) Rio de Janeiro In: O Brasil visto do espaço. EMBRAPA Informação Tecnológica, Brasília/DF, CD-Rom, 2002.

Miranda, E.E. (coord.) São Paulo In: O Brasil visto do espaço. EMBRAPA Informação Tecnológica, Brasília/DF, CD-Rom, 2002. Valmont. www.pivotvalley.com.br, 30nov2002. 